\title{
CONDITIONS IMPLYING THAT A 2-SPHERE IS ALMOST TAME
}

\author{
BY \\ L. D. LOVELAND
}

1. Introduction. Recently, Hempel [15] proved that a 2-sphere $S$ in $S^{3}$ is tame if $S$ is free and satisfies an additional Condition (A). It is not known whether $S$ is tame if it is free; however, each complementary domain of a free 2-sphere must be an open 3-cell [18]. We show that $S$ has at most two wild points if $S$ satisfies (A) and each component of $S^{3}-S$ is an open 3-cell. Thus it appears that Hempel needed the full force of freeness only to rid $S$ of two wild points.

Burgess [11] proved that a 2-sphere $S$ in $S^{3}$ is tame modulo two points if each component of $S^{3}-S$ is an open 3-cell and $S$ can be locally peripherally collared. In the next section we define a surface to be locally annular if it satisfies certain conditions more general than those in Burgess' definition of locally peripherally collared. We show in $\S 5$ that a surface is locally annular if it satisfies Hempel's Condition (A). Furthermore Burgess' result remains valid with "locally annular" replacing "locally peripherally collared" (see \$4).

No examples were given in [15] of surfaces which fail to satisfy Condition (A). It follows from our results in $\$ 5$ that the examples described in [1] and [13] each fail to satisfy (A). These examples also fail to be locally annular.

We show that a closed connected 2-manifold in $S^{3}$ is tame if it is free and locally annular (see \$6). All the main results of [15] follow as corollaries, and in addition we remove the condition in the hypothesis of Theorem 4 of [15] that the 2-manifold $M$ be a 2-sphere. In $\$ 3$ we develop a characterization of tame surfaces in $S^{3}$ which turns out to be useful in both $\S \S 4$ and 6 .

2. Definitions and notation. If $\varepsilon$ is a positive number we use " $\varepsilon$-disk", " $\varepsilon$-set", " $\varepsilon$-annulus", etc., to mean that the given set has diameter less than $\varepsilon$. However, when we say $f$ is an $\varepsilon$-map we mean that $d(x, f(x))$ is less than $\varepsilon$ for all $x$ in the domain of the continuous function $f$. An open disk is any set homeomorphic to the interior of a circle in the plane. An open annulus is a set homeomorphic to $\left\{(x, y) \mid \frac{1}{2}<x^{2}+y^{2}<1\right\}$. We use $N(x, \varepsilon)$ to denote the set of all points within a distance $\varepsilon$ of the point $x$, and we use $N(M, \varepsilon)$ to denote the set of all points within a distance $\varepsilon$ of some point of the point set $M$.

In the following definitions we consider $M$ to be a closed connected 2-manifold in $S^{3}, U$ to be a component of $S^{3}-M$, and $p$ to be a point of $M$.

Condition (A). We say that $M$ satisfies Condition (A) provided that whenever

Presented to the Society, November 4, 1966 under the title Conditions which imply that a free surface is tame; received by the editors January 4, 1967. 
$D$ is a polyhedral disk in $S^{3}$ with $\mathrm{Bd} D$ in $S^{3}-M$ and $V$ is any neighborhood of $D$ there is a disk $D^{\prime}$ (not necessarily tame) such that

(i) $\operatorname{Bd} D=\operatorname{Bd} D^{\prime}$,

(ii) $D^{\prime} \subset V$, and

(iii) if $C$ is the component of $D^{\prime}-M$ which contains $\mathrm{Bd} D^{\prime}$, then $D^{\prime}-C$ has only a finite number of components.

Also $M$ satisfies Condition (A) relative to $U$ if the above definition holds for those disks $D$ with $\mathrm{Bd} D$ in $U$.

A simple closed curve $J$ is said to be essential on an annulus (or on an open annulus) $A$ provided $J$ lies on $A$ and bounds no disk on $A$. We say that a simple closed curve $J$ spans $M$ if and only if $J \cap M$ consists of two points and $J$ intersects each component of $S^{3}-M$.

Locally AnNular. We say that $M$ is locally annular in $U$ at $p$ if and only if for each $\varepsilon>0$ and for each simple closed curve $J$ which spans $M$ and contains $p$, there is an open annulus $A$ in $U \cap N(p, \varepsilon)$ such that

(i) $J \cap \bar{A}=\varnothing$,

(ii) one component of $\mathrm{Bd} A$ is a simple closed curve $K$ in $U$ so that $J$ links $K$, and

(iii) $\mathrm{Bd} A-K \subset M$.

In case the above definition holds at each point of $M$ we say $M$ is locally annular in $U$. If the above definition holds for each point $p$ of $M$ and for each component $U$ of $S^{3}-M$ we say that $M$ is locally annular.

The above definition is similar to what is meant by $M$ being locally peripherally collared from $U$ at $p$ [11]. The major difference is that in [11] the annulus $A$ has two simple closed curves as its boundary components. Thus a locally peripherally collared surface is locally annular. In $\$ 4$ of this paper we modify the proofs in [11] to get similar results for locally annular surfaces.

We say that $M$ is free relative to $U$ if and only if for each $\varepsilon>0$ there is an $\varepsilon$-map of $M$ into $U$. Then $M$ is free in $S^{3}$ if and only if $M$ is free relative to each complementary domain of $M$.

The manifold $M$ is tame in $S^{3}$ if there is a homeomorphism of $S^{3}$ onto itself which takes $M$ onto a polyhedron. Also $M$ is tame from $U$ if $M \cup U$ is a 3-manifoldwith-boundary. We define $M$ to be locally tame from $U$ at $p$ if $p$ lies in a subset $V$ of $M \cup U$ such that $V$ is open relative to $M \cup U$ and the closure of $V$ is a topological cube. It follows from [2], [3], and [17] that $M$ is tame if and only if $M$ is tame from each component of $S^{3}-M$; and that $M$ is tame from $U$ if and only if $M$ is locally tame from $U$ at each point of $M$. If $K$ is a subset of $M$ we say that $M$ is locally tame from $U$ modulo $K$ if and only if $M$ is locally tame from $U$ at each point of $M-K$.

For other definitions see [4], [10], or [15].

3. A characterization of tame surfaces. Let $M$ be a closed connected 2-manifold in $S^{3}$, let $U$ be a component of $S^{3}-M$, and let $p$ be a point of $M$. We say that $M$ can be locally $\alpha$-capped from $U$ at $p$ if and only if for each $\varepsilon>0$ there is an $\varepsilon$-disk 
$R$ on $M$ so that $p \in$ Int $R$ and for each $\alpha>0$ there is an open $\varepsilon$-disk $D$ in $U \cap N(M, \alpha)$ such that $\mathrm{Bd} D$ lies in $M-R$ and $R$ lies on the boundary of an $\varepsilon$-component of $U-D$. If the above definition holds for each $p$ in $M$ we say that $M$ can be locally $\alpha$-capped from $U$; and if the above definition is independent of both $p$ and $U$ we say that $M$ can be locally $\alpha$-capped.

THEOREM 1. If $M$ can be locally $\alpha$-capped from $U$ at $p \in M$, then $U$ is locally simply connected at $p$.

Proof. Let $N$ be an open set containing $p$, and choose a positive number $\varepsilon$ such that $2 \varepsilon<d\left(p, S^{3}-N\right)$. Let $R$ be an $\varepsilon$-disk on $M$ such that $p \in$ Int $R$ and for each $\alpha>0$ there exists an open $\varepsilon$-disk $D$ in $U \cap N(M, \alpha)$ where $\operatorname{Bd} D$ lies in $M-R$ and $R$ lies on the boundary of an $\varepsilon$-component of $U-D$. Such a disk $D$ will lie in $N$.

Let $V$ be an open set such that $p \in V$, Bd $V$ is a tame 2-sphere, and $\bar{V} \cap M$ lies in Int $R$, and let $f$ be a map of $R$ into $V$ such that $f(\mathrm{Bd} R) \subset U$. Choose a positive number $\alpha$ such that $\alpha<d(M, f(\operatorname{Bd} R))$, and let $D$ be an open $\varepsilon$-disk chosen as above relative to this $\alpha$. Let $C$ be the $\varepsilon$-component of $U-D$ having $R$ on its boundary.

We shall show that $f \mid \mathrm{Bd} R$ can be extended to map $R$ into $U \cap N$. This will show that $U$ is locally simply connected at $p$.

Now $D$ separates $f(\operatorname{Bd} R)$ from $f(R) \cap M$ on $f(R)$ (for otherwise an $\operatorname{arc} A$ in $f(R)-D$ from a point in $f(\operatorname{Bd} R)$ to a point in $R$ would lie in $\bar{C}$, contrary to the choice of $\alpha$ ). Let $Q$ be the component of $R-f^{-1}(D)$ containing $\operatorname{Bd} R$. Then $f(Q) \subset N \cap U$ and $f \mid Q$ can be extended to a map of $R$ into $f(Q) \cup D$ which lies in $N \cap U$.

THEOREM 2. If $V$ is an open subset of $M$ so that $M$ can be locally $\alpha$-capped from $U$ at each point of $V$, then $M$ is locally tame from $U$ at each point of $V$.

Proof. From Theorem $1, U$ is locally simply connected at each point of $V$. Thus Theorem 2 follows from the proof of Theorem 4 of [10].

THEOREM 3. If $M$ can be locally $\alpha$-capped, then $M$ is tame.

Proof. It follows from Theorem 1 that each component of $S^{3}-M$ is locally simply connected at each point of $M$. Thus each such component is 1-ULC, so Theorem 3 follows from [4].

REMARK. We define $M$ to be locally capped if and only if for each $\varepsilon>0$, for each $p \in M$, and for each component $U$ of $S^{3}-M$ there exists an $\varepsilon$-disk $R$ on $M$ and an $\varepsilon$-open disk $D$ in $U$ such that

(1) $p \in$ Int $R$,

(2) $\mathrm{Bd} D \subset M-R$, and

(3) $R$ lies on the boundary of an $\varepsilon$-component of $U-D$.

We were unable to answer the following

QUESTION. Is a closed connected 2-manifold $M$ tame if it can be locally capped?

Results related to this question can be found in [4], [10], and [16]. 
4. Sufficient conditions for 2 -spheres to be tame modulo two points. The next two lemmas are used in several places in the remainder of the paper in connection with the definition of locally annular.

LEMmA 1. Suppose $S$ is a 2-sphere in $S^{3}, U$ is a component of $S^{3}-S, E$ is a disk on $S, J$ is a simple closed curve such that $J \cap E$ is a single point $p, A$ is an open annulus in $U$ such that one component of $\mathrm{Bd} A$ is a simple closed curve $K$ in $U, J$ links $K, J \cap \bar{A}=\varnothing$, and $\mathrm{Bd} A-K \subset E$. Then $\mathrm{Bd} A-K$ separates $J \cap S-\{p\}$ from $p$ on $S$.

Proof. Let us denote $S$-Int $E$ by $B$. It will be sufficient to prove that $\mathrm{Bd} A-K$ separates $p$ from $B$ on $S$. Suppose this is not the case. Then there exists an arc $H$ from $p$ to a point in $B$ such that $H \cap \bar{A}=\varnothing$. Let $r$ be a retraction of $S \cup$ ( $U$ minus a point) onto $S$ [9]. Now there exists a simple closed curve $K^{\prime}$ essential in $A$ such that the identity map on $K^{\prime}$ is homotopic to $r \mid K^{\prime}$ in $S^{3}-(J \cup H \cup B)$. Since $r\left(K^{\prime}\right) \subset E-H, r \mid K^{\prime}$ is null homotopic in $E-H$ and $K$ can be shrunk to a point in $S^{3}-J$. This is a contradiction since $J$ links $K$.

LEMMA 2. Suppose we have the same conditions as in Lemma 1 and $J^{\prime}$ is a simple closed curve containing a subarc that pierces $E$ at $p$ such that $J^{\prime} \cap E=\{p\}$ and $J^{\prime} \cap \bar{A}=\varnothing$; then $K$ cannot be shrunk to a point in the complement of $J^{\prime}$.

Proof. As in the proof of Lemma 1 there exists a loop $f$ homotopic in $S^{3}-\left(J \cup J^{\prime}\right)$ to the identity map on $K$. If $K$ can be shrunk to a point in $S^{3}-J^{\prime}$, then $f$ can also be contracted there. But $f$ is homotopic in $S^{3}-J^{\prime}$ to some odd multiple of $\mathrm{Bd} E$, so we have the contradiction that some odd multiple of Bd $E$ can be contracted in $S^{3}-J^{\prime}$.

THEOREM 4. If $C$ is a cellular crumpled cube in $S^{3}$ and $V$ is an open subset of $\mathrm{Bd} C$ such that $\mathrm{Bd} C$ is locally annular in $S^{3}-C$ at each point of $V$, then there is a point $p$ in $V$ such that $\mathrm{Bd} C$ is locally tame from $S^{3}-C$ at each point of $V-\{p\}$.

Proof. We let $S$ denote Bd $C$. If $S$ can be locally $\alpha$-capped from $S^{3}-C$ at each point of $V$, then Theorem 4 follows from Theorem 2 . Otherwise there is a point $p_{1}$ of $V$ so that $S$ cannot be locally $\alpha$-capped from $S^{3}-C$ at $p_{1}$. We will show that under these circumstances $S$ can be locally $\alpha$-capped from $S^{3}-C$ at each point of $V-\left\{p_{1}\right\}$. Then Theorem 4 will also follow from Theorem 2 in this case.

Let $p_{2}$ be a point of $V$ different from $p_{1}$, let $\varepsilon>0$ be given, and let $\delta$ be a positive number such that

(1) $7 \delta<\varepsilon$.

Let $J$ be a simple closed curve which spans $S$ and intersects $S$ at $\left\{p_{1}, p_{2}\right\}$. Using Lemma 1 and the hypothesis that $S$ is locally annular in $S^{3}-C$ at $p_{1}$ and $p_{2}$, we obtain two disjoint open annuli $A_{1}$ and $A_{2}$, two simple closed curves $J_{1}$ and $J_{2}$, and two open sets $D_{1}$ and $D_{2}$ such that for each $i$,

(2) $J_{i}$ is a boundary component of $A_{i}$ in $S^{3}-C$,

(3) $J$ links $J_{i}$, 
(4) $D_{i}$ is the component of $S-\mathrm{Bd} A_{i}$ containing $p_{i}$,

(5) $A_{i} \subset S^{3}-C$, and

(6) $S \cap \bar{A}_{i}$ lies in a disk $E_{i}$ on $S$ so that $D_{i} \subset E_{i}$ and diam $\left(E_{i} \cup A_{i}\right)<\delta$.

Let $R_{1}$ and $R_{2}$ be disjoint disks in $S$ so that $p_{i}$ is in Int $R_{i}$ and $R_{i} \subset D_{i}$, for each $i$. Then each $R_{i}$ is an $\varepsilon$-disk which does not intersect $\mathrm{Bd} A_{i}$. We will eventually show that $R_{2}$ can be used as the " $R$ " in the definition of "locally $\alpha$-capped from $S^{3}-C$ at $p_{2}$ ". Thus we assume $\alpha$ to be a given positive number.

For each $i(i=1,2)$ we let $X_{i}$ be an arc in $J-\operatorname{Int} C$ from $p_{i}$ to a point $t_{i}$ such that $X_{1} \cap X_{2}=\varnothing$ and $\operatorname{diam}\left(X_{i}\right)<\delta$. With no loss in generality we assume that

(7) $\alpha<\delta$,

(8) $\alpha<d\left(S,\left\{t_{1}, t_{2}\right\}\right)$, and

(9) $\alpha<d\left(S, J_{1} \cup J_{2}\right)$.

Since $C$ is cellular there is a 2 -sphere $S_{1}$ in $S^{3}-C$ such that

(10) $S_{1} \subset N(S, \alpha)$.

From (8), (9), and (10) we see that $J_{1} \cup J_{2} \cup\left\{t_{1}, t_{2}\right\}$ and $S$ are in different components of $S^{3}-S_{1}$.

Let $\sigma$ be a positive number chosen so small that

(11) $\sigma<\alpha$ and

(12) $N(S, \sigma) \cap S_{1}=\varnothing$.

Let $A_{1}^{\prime}$ and $A_{2}^{\prime}$ be open annuli in $A_{1}$ and $A_{2}$, respectively, such that

(13) $A_{i}^{\prime} \subset N(S, \sigma)$ and

(14) the boundary of $A_{i}-A_{i}^{\prime}$ consists of two simple closed curves $J_{i}$ and $J_{i}^{\prime}$.

We see from (12) and (13) that $S \cup A_{1}^{\prime} \cup A_{2}^{\prime}$ and $J_{1} \cup J_{2}$ lie in different components of $S^{3}-S_{1}$.

Let $S_{2}$ be a 2 -sphere such that $S$ and $S_{1} \cup\left(A_{1}-A_{1}^{\prime}\right) \cup\left(A_{2}-A_{2}^{\prime}\right)$ are in different components of $S^{3}-S_{2}$ and

(15) $S_{2} \subset N(S, \sigma)$.

Using [5] we may assume that each $A_{i}^{\prime}$ is locally polyhedral and in general position with respect to $S_{2}$ so that each component of $S_{2} \cap\left(A_{1} \cup A_{2}\right)$ is a simple closed curve and there are only finitely many such components. Because $S_{2}$ separates $S$ from $A_{i}-A_{i}^{\prime}$ there must be an essential simple closed curve in each $S_{2} \cap A_{i}^{\prime}$. We choose a disk $D^{\prime}$ in $S_{2}$ so that Bd $D^{\prime}$ is an essential simple closed curve on either $A_{1}^{\prime}$ or $A_{2}^{\prime}$ and there is no simple closed curve in Int $D^{\prime}$ which is essential on either $A_{1}^{\prime}$ or $A_{2}^{\prime}$.

The remainder of the proof is devoted to showing that if $\mathrm{Bd} D^{\prime}$ lies on $A_{i}^{\prime}$ then Bd $C$ can be locally $\alpha$-capped in $S^{3}-C$ at $p_{i}$. Once this is established we are able to conclude that $\mathrm{Bd} D^{\prime}$ lies on $A_{2}^{\prime}$ since $p_{1}$ was chosen as a point where $S$ cannot be locally $\alpha$-capped from $S^{3}-C$. Thus it will follow that $S$ can be locally $\alpha$-capped from $S^{3}-C$ at $p_{2}$, which is what we set out to prove. We will assume, just to be definite, that $\mathrm{Bd} D^{\prime} \subset A_{1}^{\prime}$.

Using the usual disk replacement process $[4$, p. 297] we obtain a disk $D$ so that Bd $D=\operatorname{Bd} D^{\prime}$, 
(16) Int $D \subset S^{3}-\left(C \cup A_{1} \cup A_{2} \cup S_{1}\right)$, and

(17) $D \subset D^{\prime} \cup\left[N\left(A_{1}^{\prime} \cup A_{2}^{\prime}, \delta\right) \cap N(S, \sigma)\right]$.

In the next paragraph we show there is an arc $Y$ in $X_{1}$ from $p_{1}$ to a point $a$ in Int $D$ so that

(18) diam $Y<\delta$ and

(19) $Y \cap D=\{a\}$.

Let $Z$ be an arc in $S^{3}-$ Int $S_{1}$ from $t_{1}$ to a point $w$ of $J_{2}$ so that the interior of $Z$ does not intersect $X_{1}$, and let $W$ be an arc from $w$ to a point $v$ in $S$ so that $W$ is close enough to $A_{2}$ to insure that $W \cap\left(D_{1} \cup D\right)=Z \cap$ Int $W=\varnothing$. This is possible by (16). Let $J^{\prime \prime}$ be a simple closed curve contained in the union of $X_{1} \cup Z \cup W$ with an open arc in Int $C$ from $v$ to $p_{1}$. It follows from Lemmas 1 and 2 that neither $J_{1}^{\prime}$ nor Bd $D$ can be shrunk to a point in $S^{3}-J^{\prime \prime}$. This means that $J^{\prime \prime}$ must intersect $D$, but $J^{\prime \prime}-X_{1}$ was chosen in the complement of $D$. Thus it is apparent how to construct $Y$ in $X_{1}$.

Suppose that the diameter of $D$ is larger than $6 \delta$. We will obtain a contradiction using an argument similar to that given in [11]. There must be a point $b$ in Int $D$ so that

(20) $d(a, b) \geqq 3 \delta$.

It follows from (15), (17), and the fact that $D^{\prime} \subset S_{2}$ that there is a point $c$ of $S$ such that the line segment $c b$ has diameter less than $\sigma$. Then from (7), (11), and (12),

(21) $d(c, b)<\delta$ and

(22) $c b \cap S_{1}=\varnothing$.

From (18) and (6) it follows that $D_{1} \cup A_{1}$ lies in a $2 \delta$ neighborhood of $a$, so that from (20) and (21) we have

(23) $c b \cap\left(D_{1} \cup A_{1}\right)=\varnothing$.

Let $B$ be an arc from $a$ to $c$ such that $B$ contains $c b$ and $B-c b$ lies in $D$, and let $L$ be a simple closed curve containing $Y \cup B$ such that $L-Y \cup B \subset$ Int $C$. Then $L$ pierces $S$ at $p_{1}, L \cap\left(A_{1} \cup S_{1}\right)=\varnothing$, and $L \cap E_{1}=\left\{p_{1}\right\}$. Since $L$ and $J_{1}$ lie in different components of $S^{3}-S_{1}$ we have a contradiction to Lemma 2 .

Therefore,

(24) diam $D<6 \delta$.

Now one component, say $F$, of $A_{1}^{\prime}-\mathrm{Bd} D$ is an open annulus with one boundary component on $S$. We let $E=D \cup F$ so that $E$ is an open disk in $S^{3}-S$ such that Bd $E \subset S-R_{1}$. It follows from (6), (24), and (1) that

(25) $\operatorname{diam} E<\varepsilon$.

All that remains in showing that $S$ can be locally $\alpha$-capped from $S^{3}-C$ at $p_{1}$ is to show that

(26) $E \subset N(S, \alpha)$ and

(27) $R_{1}$ lies on the boundary of an $\varepsilon$-component of $\left(S^{3}-C\right)-E$.

Condition (26) follows from (11), (13), (15), and (17).

Let $H$ be the component of $\left(S^{3}-C\right)-E$ such that $R_{1} \subset \mathrm{Bd} H$. Condition (27) will follow from (6), (24), and (1) once we show that $\mathrm{Bd} H \subset E_{1} \cup A_{1} \cup D$. If this 
is not the case there must be a point $q$ of $\mathrm{Bd} H$ so that $q$ is not in $E_{1} \cup A_{1} \cup D$. Then $q$ is in $S$ and there is a simple closed curve $J^{\prime}$ which spans $S$, intersects $S$ only at $p_{1}$ and $q$, and which does not intersect $F \cup D$. This contradicts Lemma 2 since $J^{\prime}$ does not intersect $D$.

COROLLARY 1. If $C$ is a cellular crumpled cube and $W$ is the set of points where $\mathrm{Bd} C$ is wild from $S^{2}-C$, then either

(1) $W=\varnothing$,

(2) $W$ is degenerate, or

(3) $W$ contains a nondegenerate continuum.

COROLlary 2. With the same hypothesis as in Corollary $1, W$ does not contain two isolated points.

COROLlary 3 (BuRgess). If $S$ is a 2-sphere in $S^{3}$ such that each component of $S^{3}-S$ is an open 3-cell and the set $W$ of wild points of $S$ is 0-dimensional, then $W$ contains at most two points.

5. Condition (A) implies that $M$ is locally annular. If $D$ is a disk and $D_{1}, D_{2}$, $\ldots, D_{n}$ is a finite collection of disjoint disks in Int $D$, then $D-\cup$ Int $D_{i}$ is called a disk-with-holes.

LEMMA 3. If $D$ is a disk-with-holes in $S^{3}$. $J$ is a simple closed curve which links one component of $\mathrm{Bd} D$, and $J$ does not intersect $D$, then $J$ links at least two components of $\mathrm{Bd} D$.

Proof. The proof $f_{0}$ !nows by repeated use of Theorems 9 and 10 of [5].

THEOREM 5. If $C$ is a crumpled cube in $S^{3}$ such that $\mathrm{Bd} C$ satisfies Condition (A) relative to $S^{3}-C$, then $\mathrm{Bd} C$ is locally annular in $S^{3}-C$.

Proof. We will let $S$ denote the 2-sphere that is the boundary of $C$. Let $J$ be a simple closed curve that spans $S$ such that $J \cap S$ consists of the two points $p$ and $q$, and let $\varepsilon$ be a positive number. We must show that there is an open annulus $A$ in $\left(S^{3}-C\right) \cap N(p, \varepsilon)$ such that $J \cap \bar{A}=\varnothing$, one component $K$ of $\mathrm{Bd} A$ is a simple closed curve in $U$ such that $J$ links $K$, and $\mathrm{Bd} A-K$ lies in $S$.

Let $N$ be an open $\varepsilon$-set such that $p$ lies in $N$ and $q \in S-\bar{N}$. Let $G$ be an annulus in $N \cap S$ so that each component of $\mathrm{Bd} G$ is a tame simple closed curve which separates $p$ from $q$ [8]. Let the closure of the component of $S-G$ which contains $p$ be called $R$. Then $R$ is a disk on $S$ so that $p \in \operatorname{Int} R$.

Let $\sigma$ be a positive number such that

(1) $\sigma<d(J, G)$ and

(2) $\sigma<d(G, \mathrm{Bd} N)$.

Using Bing's Side Approximation Theorem for the open set Int $G$ [6] we obtain an annulus $B$, a homeomorphism $h$ from $B$ into $G$, and a collection of disjoint $\sigma$-disks $Q_{1}, Q_{2}, Q_{3}, \ldots$ (locally finite modulo $\mathrm{Bd} R$ ) in Int $B$ such that

(3) $h$ moves no point more than a distance $\sigma$, 
(4) $B$-Int $Q_{1}-\mathrm{Bd} R \subset S^{3}-C$,

(5) $B \cap$ Int $R=\varnothing$,

(6) $\mathrm{Bd} R \subset \mathrm{Bd} B$, and

(7) $B-\mathrm{Bd} R$ is locally polyhedral.

Let $F=\mathrm{Bd} B-\mathrm{Bd} R$. It follows from (1), (6), and (3) that the identity maps on $F$ and $\mathrm{Bd} R$ are homotopic in the complement of $J$; hence, from [5],

(8) $J$ links $F$.

Also from (4), (2), (3), and (6),

(9) $F \subset S^{3}-C$ and

(10) $B \subset N$.

Now we apply the Side Approximation Theorem again, this time relative to the open set Int $R$, to obtain a disk $E$ "almost in Int $C$ " (in the sense of (4)) such that $\mathrm{Bd} E=\mathrm{Bd} R, E \subset N$, and $E \cap B=\mathrm{Bd} R$. Then $E \cup B$ is a disk $D^{\prime}$ which is locally polyhedral modulo the tame simple closed curve $\mathrm{Bd} R$. Since $D^{\prime}$ is tame [7], [12] we assume with no loss in generality that $D^{\prime}$ is polyhedral.

Let $V$ be an open set such that $D^{\prime} \subset V$ and the closure $X$ of the component of $V-S$ containing Bd $D^{\prime}$ does not intersect $J$. From condition (A) there is a disk $D$ in $V$ such that

(11) $\mathrm{Bd} D=\mathrm{Bd} D^{\prime}$,

(12) $D \subset V$, and

(13) the component $T$ of $D-S$ which contains $\mathrm{Bd} D$ has the property that $D-T$ has only a finite number of components.

As in the proof of Theorem 2 of [15] we choose a finite collection of disjoint subdisks $D_{1}, D_{2}, \ldots, D_{n}$ of $D$ so that, for each $i$, the boundary $J_{i}$ of $D_{i}$ lies in $T$ and the interior of the component $A_{i}$ of $D_{i}-S$ which contains $J_{i}$ is an open annulus in $S^{3}-C$. It is Condition (13) that makes the interior of $A_{i}$ an open annulus. Now $D-\cup$ Int $D_{i}$ is a disk-with-holes and $J$ links Bd $D$ from (8), so it follows from Lemma 3 that $J$ links some $J_{j}$. From (12) and the fact that $V \subset N$, we see that Int $A_{j}$ is an open $\varepsilon$-annulus.

Since $\bar{A}_{j} \subset \bar{T} \subset X$, it follows that $\bar{A}_{j} \cap J=\varnothing$. Now the open annulus Int $A_{j}$ fits the definition of $S$ being locally annular in $S^{3}-C$ at $p$. Because $p$ was chosen as an arbitrary point of $S, S$ is locally annular in $S^{3}-C$.

THEOREM 6. If $M$ is a closed connected 2-manifold in $S^{3}, U$ is a component of $S^{3}-M$, and $M$ satisfies Condition (A) relative to $U$, then $M$ is locally annular in $U$.

Proof. Let $p \in M$. Using Theorem 5 of [4] we obtain a disk $D$ in $M$ and a 2sphere $S$ such that $p \in \operatorname{Int} D \subset S$. One component $V$ of $S^{3}-S$ has the property that $D$ lies on the boundary of $V \cap U$. Now relative to any simple closed curve $J$ which spans $M$ and contains $p$ we are able to apply the argument of the proof of Theorem 5 to show that $S$ is locally annular in $V$ at $p$. This implies that $M$ is locally annular in $U$ at $p$.

THEOREM 7. If a cellular crumpled cube $C$ in $S^{3}$ has a boundary which satisfies 
Condition (A) relative to $S^{3}-C$, then there is a point $p$ in $\mathrm{Bd} C$ so that $\mathrm{Bd} C$ is locally tame from $S^{3}-C$ modulo $\{p\}$.

Proof. It follows from Theorem 5 that $\mathrm{Bd} C$ is locally annular in $S^{3}-C$. Thus Theorem 7 follows from Theorem 4 .

COROLlARY 4. If $S$ is a 2-sphere in $S^{3}$ such that each component of $S^{3}-S$ is an open 3-cell and $S$ satisfies Condition (A), then $S$ has at most two wild points.

CoRollary 5. If C is a cellular 3-cell in $S^{3}$ such that $\mathrm{Bd} C$ satisfies Condition (A), then $\mathrm{Bd} C$ is locally tame modulo a point.

REMARK. No examples were given in [15] of surfaces which fail to satisfy Condition (A). It follows from Corollary 5 that the examples in [1] and [13] fail to satisfy (A); also neither of these examples is locally annular. The following question remains open:

QuESTION. If a closed connected 2-manifold $M$ in $S^{3}$ is free relative to a component $U$ of $S^{3}-M$, then does $M$ satisfy Condition (A) relative to $U$ ?

An answer to this question would also provide an answer to the question raised on p. 280 of [14].

6. A 2-manifold is tame if it is locally free and locally annular. Throughout this section we let $M$ denote a closed connected 2-manifold in $S^{3}$, we let $U$ denote a component of $S^{3}-M$, and we let $p$ be a point of $M$. We say that $M$ is locally free in $U$ at $p$ if and only if there is a disk $G$ in $M$ so that $p \in \operatorname{Int} G$ and for each $\varepsilon>0$ there is an $\varepsilon$-map of $G$ into $U$. Also $M$ is locally free in $U$ if the above definition is independent of the point $p$ in $M$, and $M$ is locally free if $M$ is locally free in each component of $S^{3}-M$.

Hempel's proof of Theorem 2 of [15] needs only slight modification to establish the following result.

TheOREM 8 (Hempel). If $M$ is locally free in $U$ and $M$ satisfies Condition (A) relative to $U$, then $M$ is tame from $U$.

We show in Theorem 12 that Theorem 8 remains valid if " $M$ is locally annular in $U$ " is substituted for " $M$ satisfies Condition (A) relative to $U$ " in the hypothesis. Then the results of $\S \S 4$ and 5 of [15] follow as corollaries. Also we obtain Hempel's Theorem 4 without the restriction that the closed connected 2-manifold $M$ be a 2-sphere (see Corollary 7).

We begin by outlining a proof for Theorem 9 although it is a corollary to Theorem 12 which follows. The reason for including this special case separately is that there is an interesting short proof based on Theorem 2 of [15]. Corollary 6 is Theorem 4 of [15].

THEOREM 9. If a 2-sphere $S$ in $S^{3}$ is free relative to a component $U$ of $S^{3}-S$ and $S$ is locally annular in $U$, then $S$ is tame from $U$. 
Proof. Using the hypothesis that $S$ is free relative to $U$ together with the Sphere Theorem [18] we see that $S^{3}-U$ is cellular. Then it follows from Theorem 4 that $S$ is locally tame from $U$ except at possibly one point. This means that $S$ satisfies Condition (A) relative to $U$, so it follows from Theorem 2 of [15] that $S$ is tame from $U$.

COROLlARY 6 (HEMPEL). If a 2-sphere $S$ in $S^{3}$ is locally tame from a component $U$ of $S^{3}-S$ modulo a 0 -dimensional set and $S$ is free relative to $U$, then $S$ is tame from $U$.

THEOREM 10. If $M$ is locally free in $U$ at $p$ and $M$ is locally annular in $U$, then $U$ is locally simply connected at $p$.

Proof. Let $J$ be a simple closed curve that spans $M$ such that $J \cap M$ consists of the two points $p$ and $q$. Since $M$ is locally free in $U$ at $p$, there is a disk $G$ on $M$ such that $p \in \operatorname{Int} G, q \in M-G$, and for each $\eta>0$ there is an $\eta$-map of $G$ into $U$. We may assume without loss in generality that $G$ lies on a 2-sphere $S$ such that $G$ lies on the boundary of the intersection of $U$ with one component, which we call Int $S$, of $S^{3}-S$ [4, Theorem 5]. We shall show that $S$ can be locally $\alpha$-capped in Int $S$ at $p$ so that it will follow from Theorem 1 that Int $S$ is locally simply connected at $p$. This will imply that $U$ is locally simply connected at $p$ since $G \subset \operatorname{Bd}(U \cap \operatorname{Int} S)$.

Let $\varepsilon>0$ be given, and let $E$ be a disk such that $p \in \operatorname{Int} E \subset E \subset \operatorname{Int} G$ and diam $E$ $<\varepsilon / 3$. As in the definition of "locally annular", let $A$ be an open annulus in $U \cap$ Int $S \cap N(p, \varepsilon)$ such that $J \cap \bar{A}=\varnothing$, one component of $\mathrm{Bd} A$ is a simple closed curve $K$ that links $J$, and $\mathrm{Bd} A-K$ lies in Int $E$. We also assume that $A$ is locally polyhedral [5]. Following the definition of "locally $\alpha$-capped" we identify a disk $R$ on $S$ such that $p \in \operatorname{Int} R, R \subset \operatorname{Int} E$, and $R \cap \bar{A}=\varnothing$; and we suppose that $\alpha$ is a given positive number. We shall indicate how to obtain an open $\varepsilon$-disk $D$ in $N(E, \alpha) \cap$ Int $S$ such that $R$ lies on the boundary of an $\varepsilon$-component of Int $S-D$.

In the annulus $A$ we choose a subannulus $A_{1}$ with boundary components Bd $A-K$ and a simple closed curve $J_{1}$ such that diam $A_{1}<\alpha$ and $A_{1} \subset N(E, \alpha)$. Now a proof much like Hempel gave for Theorem 2 of [15] shows the existence of a disk $E_{1}$ in Int $E$ and a map $F_{1}$ such that $F_{1}$ takes Bd $E_{1}$ essentially into $A_{1}$, $F_{1}\left(E_{1}\right)=D_{1}$ has diameter less than $\varepsilon / 3$, and $D_{1} \subset U$. Little additional argument is needed to show that $D_{1}$ can be chosen in $N(E, \alpha)$.

Using Dehn's Lemma [18] relative to a singular disk in $A_{1} \cup D_{1}$ having a nonsingular neighborhood of its boundary and containing $D_{1}$, we obtain the desired disk $D$. An argument like that given in the last paragraph of the proof of Theorem 4 shows that the component of Int $S-D$ whose boundary contains $R$ has small diameter.

THEOREM 11. If $V$ is an open subset of $M$ such that $M$ is locally free in $U$ at each point of $V$ and $M$ is locally annular in $U$ at each point of $V$, then $M$ is locally tame from $U$ at each point of $V$. 
Proof. From Theorem 10 it follows that $U$ is locally simply connected at each point of $V$. Then Theorem 11 follows from the proof of Theorem 4 of [10].

THEOREM 12. If $M$ is locally free in $U$ and locally annular in $U$, then $M$ is tame from $U$.

Proof. If we apply Theorem 11 , where $V=M$, we see that $M$ is locally tame from $U$. Then $M$ is tame from $U$ [2], [17].

COROLLARY 7. If $M$ is locally free in $U$ and $M$ is locally tame from $U$ modulo a 0-dimensional set, then $M$ is tame from $U$.

COROLlaRy 8. If $M$ is locally free and locally annular, then $M$ is tame.

COROllary 9. If $M$ is locally free and locally tame modulo a 0-dimensional set, then $M$ is tame.

REMARK. Burgess has defined " $M$ is locally peripherally collared" to mean that for each $\varepsilon>0$, for each point $p \in M$, and for each component $U$ of $S^{3}-M$, there is a disk $D$ and an annulus $A$ such that $p \in \operatorname{Int} D \subset M$, diam $D<\varepsilon, \operatorname{Bd} D \subset \operatorname{Bd} A$, and $A-\operatorname{Bd} D \subset U$. Since a 2-manifold satisfying this condition is locally annular we have the following result.

COROLlaRY 10. If $M$ is locally free and locally peripherally collared, then $M$ is tame.

\section{REFERENCES}

1. W. R. Alford, "Some "nice" wild 2-spheres in $E^{3}$," in Topology of 3-manifolds and related topics, pp. 29-33, Prentice-Hall, Englewood Cliffs, N. J., 1962.

2. R. H. Bing, Locally tame sets are tame, Ann. of Math. (2) 59 (1954), 145-158.

3. - An alternative proof that 3-manifolds can be triangulated, Ann. of Math. (2) 69 (1959), 37-65.

4. - A surface is tame if its complement is 1-ULC, Trans. Amer. Math. Soc. 101 (1961), 294-305.

5. - Approximating surfaces with polyhedral ones, Ann. of Math. (2) 65 (1957), 456-483.

6. _-, Approximating surfaces from the side, Ann. of Math. (2) 77 (1963), 145-192.

7. - Pushing a 2-sphere into its complement, Michigan Math. J. 11 (1964), 33-45.

8. - Each disk in $E^{3}$ contains a tame arc, Amer. J. Math. 84 (1962), 583-590.

9. - Retractions onto spheres, Amer. Math. Monthly 71 (1964), 481-484.

10. C. E. Burgess, Characterizations of tame surfaces in $E^{3}$, Trans. Amer. Math. Soc. 114 (1965), 80-97.

11. - Criteria for a 2-sphere in $S^{3}$ to be tame modulo two points, Michigan Math. J. 14 (1967), 321-330.

12. P. H. Doyle and J. G. Hocking, Some results on tame disks and spheres in $E^{3}$, Proc. Amer. Math. Soc. 11 (1960), 832-836.

13. D. S. Gillman, Note concerning a wild sphere of Bing, Duke Math. J. 31 (1964), 247-254.

14. John Hempel, $A$ surface in $S^{3}$ is tame if it can be deformed into each complementary domain, Trans. Amer. Math. Soc. 111 (1964), 273-287.

15. - Free surfaces in $S^{3}$, (to appear). 
16. L. D. Loveland, Tame surfaces and tame subsets of spheres in $E^{3}$, Trans. Amer. Math. Soc. 123 (1966), 355-368.

17. E. E. Moise, Affine structures in 3-manifolds. VIII. Invariance of the knot-types; local tame inbedding, Ann. of Math. (2) 59 (1954), 169-170.

18. C. D. Papakyrakopoulos, On Dehn's lemma and the asphericity of knots, Ann. of Math. (2) 66 (1957), 1-26.

19. — On solid tori, Proc. London Math. Soc. (3) 7 (1957), 281-299.

\section{Utah State University, LOGAN, UTAH}

